\title{
An empirical calibration of sulphur abundance in ionised gaseous nebulae
}

\author{
E. Pérez-Montero ${ }^{1}$, A. I. Díaz ${ }^{1}$, J. M. Vílchez ${ }^{2}$, and C. Kehrig ${ }^{2,3}$ \\ 1 Departamento de Física Teórica, C-XI, Universidad Autónoma de Madrid, 28049, Cantoblanco, Madrid, Spain \\ e-mail: [enrique.perez; angeles.diaz]@uam.es \\ 2 Instituto de Astrofísica de Andalucía (CSIC) Apartado de Correos 3004. 18080, Granada, Spain \\ e-mail: [jvm;kehrig]@iaa.es \\ 3 Observatório Nacional, Rua José Cristino, 77, 20.921-400, Rio de Janeiro - RJ, Brazil \\ e-mail: kehrig@on.br
}

Received 16 September 2005 / Accepted 2 November 2005

\section{ABSTRACT}

We have derived an empirical calibration of the abundance of $\mathrm{S} / \mathrm{H}$ as a function of the $\mathrm{S}_{23}$ parameter, defined using the bright sulphur lines of [SII] and [SIII]. Contrary to the case for the widely used $\mathrm{O}_{23}$ parameter, the calibration remains single valued up to the abundance values observed in the disk HII regions. The calibration is based on a large sample of nebulae for which direct determinations of electron temperatures exist and the sulphur chemical abundances can be directly derived. ICFs, as derived from the [SIV] $10.52 \mu$ emission line (ISO observations), are shown to be well reproduced by Barker's formula for a value of $\alpha=2.5$. Only about $30 \%$ of the objects in the sample require ICFs larger than 1.2. The use of the proposed calibration opens the possibility of performing abundance analysis with red to IR spectroscopic data using S/H as a metallicity tracer.

Key words. ISM: abundances - ISM: HII regions

\section{Introduction}

Oxygen is the main abundance tracer in HII regions and HII galaxies, but its abundance is uncertain in those cases in which no direct determination of the electron gas temperature exists therefore requiring the use of empirical or semiempirical methods. These methods are based on the cooling properties of ionised nebulae which ultimately reflect on the relation between emission line intensities and oxygen abundance. When the cooling is dominated by oxygen, the electron temperature depends inversely on oxygen abundance. Since the intensities of collisionally excited lines depend exponentially on temperature, a relation is expected to exist between these intensities and oxygen abundances. The $\mathrm{O}_{23}$ parameter, also known as $\mathrm{R}_{23}$ and defined as the sum of the intensities of the [OII] $\lambda \lambda 3727,29 \AA$ and [OIII] $\lambda \lambda 4959,5007 \AA$ emission lines relative to $\mathrm{H} \beta$ (Pagel et al. 1979), has been widely used for these purposes.

The relation between $\mathrm{O}_{23}$ and oxygen abundance is twofold since at high metallicities, the efficiency of the oxygen as a cooling agent decreases the strength of the oxygen emission lines while at low metallicities the cooling is mainly exerted by hydrogen and the oxygen line strengths increase with metallicity. A value of $12+\log (\mathrm{O} / \mathrm{H})$ of about 8.2 divides the two different abundance regimes. Although some line ratios have been proposed in order to break this degeneracy, the fact that a large number of HII regions/HII galaxies lie right on the turnover region is of great concern.

On the other hand, the use of $\mathrm{S} / \mathrm{H}$ as an abundance tracer has been frequently overlooked. Similarly to oxygen, sulphur is an element produced in massive stars through explosive nucleosynthesis and its yield should follow that of O. Nebular $\mathrm{S} / \mathrm{H}$ abundances are therefore expected to follow $\mathrm{O} / \mathrm{H}$ and the $\mathrm{S} / \mathrm{O}$ is expected to remain constant at approximately the solar neighborhood value, $\log \mathrm{S} / \mathrm{O} \simeq-1.6$ (e.g. Lodders 2003 ; Bresolin et al. 2004). Empirical tests exploring this have been performed confirming the cosmic nucleosynthetic ratio although the results of some works suggest that this relation should be explored further, particularly at the poorly known metallicity ends: extremely metal deficient HII galaxies (i.e. very low $\mathrm{O} / \mathrm{H}$ ) and $\mathrm{HII}$ regions in the inner disk of galaxies (i.e. metal rich central parts with highest $\mathrm{O} / \mathrm{H}$ abundances).

The strong nebular lines of sulphur are analogous to those of oxygen and hence similar reasoning may be proposed regarding the use of the $S_{23}$ parameter, $S_{23}=[S I I] \lambda \lambda 6717,6731+$ [SIII] $\lambda \lambda 9069,9532) / \mathrm{H} \beta$ (Vílchez \& Esteban 1996) as an alternative abundance indicator (Díaz \& Pérez-Montero 2000), which presents several advantages over oxygen: 1) due to the 
longer wavelengths of the lines implied, its relevance as a cooling agent starts at lower temperatures (higher metallicities) which makes the relation to remain single-valued up to solar abundances; 2) their lower dependence on electron temperature renders the lines observable even at over-solar abundances; 3) the lines of both [SII] and [SIII] can be measured relative to nearby hydrogen recombination lines, thus minimizing the effect of any reddening and/or calibration uncertainties. On the negative side, [SIII] lines shift out of the far red spectral region for redshifts higher than 0.1 . Given these properties, here we reccommend the use of spectroscopy in the red-to-near infrared wavelength range in order to derive physical properties and abundances of HII regions, including the lines from [SIII] $\lambda 6312 \AA$, [SII] $\lambda 6717,31 \AA$ up to the [SIII] $\lambda 9069,9532 \AA$. These lines can constitute a convenient analogue in this wavelength range to the role played by the [OII] and [OIII] lines in the optical. In additon, within this wavelength range the effects of extinction are less severe, thus favouring the application of this calibration to the study of HII regions located in the Milky Way disk, in the inner regions of galaxies, or to star-forming galaxies/regions affected by a large amount of extinction.

In this paper, following earlier work by Christensen et al. (1997) and by Vermeij et al. (2002), we have explored the behaviour of sulphur emission lines in order to provide a useful abundance calibration of $\mathrm{S} / \mathrm{H}$ versus $\mathrm{S}_{23}$ over the whole abundance range. This empirical calibration is based on the bright sulphur lines and encompasses the whole range of abundance currently found in HII region studies. This calibration appears to be independent of both $\mathrm{O} / \mathrm{H}$ and $\mathrm{O}_{23}$, and is especially suited for observations including (only) the red to near-infrared spectral range (from [SIII] $\lambda 6312 \AA$, H $\alpha$ up to the $1 \mu$ CCD cut-off). For this wavelength regime, the sulphur lines can be easily scaled to their respective closest Balmer or Paschen recombination line, $\mathrm{H} \lambda$ - instead of to $\mathrm{H} \beta-$ and $\mathrm{S}_{23}$ can be written as follows:

$\mathrm{S}_{23}=\mathrm{S}_{23}^{\prime} x\left(\frac{\mathrm{H} \lambda}{\mathrm{H} \beta}\right)_{0}$

where $S_{23}^{\prime}$ is the scaled value of $S_{23}$ and $(H \lambda / H \beta)_{0}$ is the theoretical case $\mathrm{B}$ recombination ratio.

The calibrations can be extended further to the infrared to include the [SIV] $10.52 \mu$ line to $S_{23}$ and defines a new parameter, $S_{234}$ (Oey \& Shields 2000). However, the lack of [SIV] data makes difficult to check its reliability.

In the next section we present the sample of objects used here to establish the sulphur abundance calibration as well as all the selected objects for which reliable ISO [SIV] data have been published. Section 3 is devoted to the study of the ionisation correction factor (ICF),- not yet well stablished for sulphur -, using selected data of HII regions including [SIV] infrared line fluxes, together with predictions of photoionisation models. In Sect. 4 we present and discuss the proposed empirical calibration of $\mathrm{S} / \mathrm{H}$ versus $\mathrm{S}_{23}$ and we summarize our conclusions.

\section{The selected sample of objects and sulphur abundances}

Our sample is a combination of different emission line objects ionised by young massive stars: diffuse HII regions in the Galaxy and the Local Group, Giant Extragalactic HII regions (GEHR) and HII galaxies, and therefore does not include planetary nebulae or objects with non-thermal activity. The emission line data along with their corresponding errors have been taken from the literature for all objects except for the extremely metal-poor galaxies studied by Kniazev et al. (2003) that present the strong [SIII] emission line at $9069 \AA$. The spectra of these objects have been taken from the data base of the DR3 of the Sloan Digital Sky Survey $\left(\mathrm{SDSS}^{1}\right)$ and the line fluxes have been measured and analyzed using the task SPLOT of the software package IRAF ${ }^{2}$, following the same procedure as in Pérez-Montero \& Díaz (2003, hereinafter PMD03). We have found a good agreement between the oxygen emission lines listed by Kniazev et al. (2003) and our measurements. The reddening corrected sulphur emission line fluxes, normalized to $I(\mathrm{H} \beta)=100$, which are relevant for this work are listed in Table 1, together with their corresponding reddening constants and errors.

For all the objects of the sample, measurements of the emission lines of [SII] at $6717,31 \AA$ and of [SIII] at $9069,9532 \AA$ exist, thus allowing the simultaneous determination of the $\mathrm{S}_{23}$ parameter and the abundances of $\mathrm{S}^{+}$and $\mathrm{S}^{2+}$.

The physical conditions of the ionised gas in each sample object, including electron temperatures, electron density and sulphur abundances, have been computed from the original emission line data using the same procedures as in PMD03, based on the five-level statistical equilibrium model in the task TEMDEN and IONIC, respectively, of the software package IRAF (De Robertis et al. 1987; Shaw \& Dufour 1995). The atomic coefficients used are the same as in PMD03 (see Table 4 of that work). Electron densities are determined from the the [SII] $\lambda 6717 \AA / \lambda 6731 \AA$ line ratio. Electron temperatures have been calculated from the [SIII] $(\lambda 9069 \AA+\lambda 9532 \AA) / \lambda 6312 \AA$ line ratio for all but 44 objects of the sample for which the [OIII] $(\lambda 4959 \AA+\lambda 5007 \AA) / \lambda 4363 \AA$ line ratio has been used. For these objects, marked with ${ }^{b}$ in Table 2, a theoretical relation between [OIII] and [SIII] electron temperatures has been used:

$t([\mathrm{SIII}])=1.05 t([\mathrm{OIII}])-0.08$.

This relation is based on the grids of photo-ionisation models described in Pérez-Montero \& Díaz (2005) and differs slightly from the empirical relation found by Garnett (1992), mostly due to the introduction of the new atomic coefficients for $\mathrm{S}^{2+}$ from Tayal \& Gupta (1999).

Regarding [SII] temperatures, for those objects without the [SII] auroral lines at 4068, $4074 \AA$. we have taken the approximation $t[\mathrm{SII}] \approx t[\mathrm{OII}]$ as valid. For 124 objects of

\footnotetext{
1 The SDSS web site is http://www . sdss.org

2 IRAF is distributed by the National Optical Astronomy Observatory.
} 
Table 1. Reddening corrected line fluxes for sulphur normalized to $I(\mathrm{H} \beta)=100$ measured in spectra of SDSS low metallicity galaxies from Kniazev et al. (2003), along with their reddening constants.

\begin{tabular}{cccccc}
\hline \hline Object & {$[\mathrm{SIII}] \lambda 6312 \AA$} & {$[\mathrm{SII}] \lambda 6716 \AA$} & {$[\mathrm{SII}] \lambda 6731 \AA$} & {$[\mathrm{SIII}] \lambda 9069 \AA$} & $C(\mathrm{H} \beta)$ \\
\hline$S D S S J 0133+1342$ & $0.9 \pm 0.2$ & $9.9 \pm 0.2$ & $8.0 \pm 0.4$ & $5.8 \pm 0.4$ & 0.03 \\
$K U G 0203-100$ & - & $23.0 \pm 1.1$ & $18.6 \pm 1.1$ & $19.6 \pm 1.1$ & 0.19 \\
$H S 0822+3542$ & $1.4 \pm 0.3$ & $2.8 \pm 0.2$ & $2.2 \pm 0.2$ & $6.0 \pm 0.2$ & 0.17 \\
$I Z w 18-N W$ & $0.8 \pm 0.1$ & $2.0 \pm 0.3$ & $1.4 \pm 0.1$ & $3.9 \pm 0.3$ & 0.05 \\
$I Z w 18-S E$ & $0.5 \pm 0.2$ & $3.9 \pm 0.2$ & $2.8 \pm 0.2$ & $3.4 \pm 0.5$ & 0.12 \\
$S B S 1102+606$ & $1.6 \pm 0.3$ & $9.6 \pm 0.6$ & $6.7 \pm 0.6$ & $10.1 \pm 0.6$ & 0.15 \\
$A 1116+517$ & $1.3 \pm 0.3$ & $7.9 \pm 0.2$ & $5.3 \pm 0.2$ & $7.7 \pm 1.8$ & 0.17 \\
$S D S S J 1121+0324$ & $1.3 \pm 0.4$ & $12.2 \pm 0.8$ & $9.6 \pm 0.8$ & $4.2 \pm 0.6$ & 0.20 \\
$S D S S J 1201+0211$ & $1.0 \pm 0.2$ & $5.4 \pm 0.2$ & $3.7 \pm 0.2$ & $5.0 \pm 0.3$ & 0.14 \\
$C G C G 269-049$ & $1.4 \pm 0.3$ & $6.5 \pm 0.3$ & $3.1 \pm 0.3$ & $5.9 \pm 0.7$ & 0.10 \\
\hline
\end{tabular}

the sample it has been possible to derive $t[\mathrm{OII}]$ from the [OII] $(\lambda 3726 \AA+\lambda 3729 \AA) / \lambda 7325 \AA$ line ratio $^{3}$. For the rest of the objects of the sample, marked with ${ }^{a}$ in Table 2, not presenting any auroral line in the low excitation zone, we have resorted to the model predicted relations between $t([\mathrm{OII}])$ and $t([\mathrm{OIII}])$ found in PMD03 that explicitly take into account the dependence of $t([\mathrm{OII}])$ on electron density. This can affect the deduced abundances of $\mathrm{S}^{+} / \mathrm{H}^{+}$by non-negligible factors, larger in all cases than the reported errors.

For those objects for which multiple observations exist we have considered each of them as independent. The final quoted errors in the derived quantities have been calculated by propagating the measurement errors in the emission lines provided by the different authors. This information is not provided for the objects from Dennefeld \& Stasińska (1983; reference 4). The ionic abundances of sulphur, $\mathrm{S}^{+} / \mathrm{H}^{+}$and $\mathrm{S}^{2+} / \mathrm{H}^{+}$, together with the values of the $S_{23}$ parameter for each object are given in Table 2.

This database include objects covering the entire abundance range from low metallicity HII galaxies, at $1 / 40$ of the solar abundance, up to HII regions in the disks of spirals populating the high metallicity range, up to $\approx 3 Z_{\odot}$. Although the data selected for this study have been obtained using different apertures and instrumental configurations and therefore do not constitute a homogeneous sample, they have been reanalysed and ionic abundances have been derived in a homogeneous manner. Using this sample we can study on a firmer basis the empirical relationship between the sulphur abundance and the parameter $S_{23}$. In order to do this we explore first the sum of the abundances of $\mathrm{S}^{+}$and $\mathrm{S}^{2+}$ as a function of $\mathrm{S}_{23}$ and then derive the calibration of the total abundance of sulphur as a function of $S_{23}$. The result is plotted in Fig. 1, showing a

\footnotetext{
3 The [OII] $\lambda 7319 \AA+\lambda 7330 \AA$ lines can have a contribution by direct recombination which increases with temperature. Using the calculated [OIII] electron temperatures, we have estimated these contributions to be less than $4 \%$ in all cases and therefore we have not corrected for this effect.
}

relationship with very low scatter for which we have obtained the following quadratic fit:

$$
12+\log \left(\frac{\mathrm{S}^{+}+\mathrm{S}^{2+}}{\mathrm{H}^{+}}\right)=6.540+2.071 \log \mathrm{S}_{23}+0.348\left(\log \mathrm{S}_{23}\right)^{2}
$$

with a typical dispersion of 0.17 dex, defined as the standard deviation of the residuals of the points.

Since ISO mission, it has also been possible to obtain data of the [SIV] at $10.52 \mu$ emission line for a selected sample of objects, thus allowing the derivation of the $\mathrm{S}^{3+} / \mathrm{H}^{+}$ionic abundance ratio. We have collected data for $11 \mathrm{HII}$ regions (plus one supernova remnant) in the Large and Small Magellanic Clouds (Vermeij et al. 2002) and for the HII galaxy Mrk 209 (Nollenberg et al. 2002). For all these objects the $\mathrm{S}^{3+}$ ionic abundances have been recalculated following the procedure described above and using the most recent atomic coefficients from Saraph \& Storey (1999). The calculated abundances are listed in Table 3.

The exact contribution of the $\mathrm{S}^{3+} / \mathrm{H}^{+}$ionic abundance to the total abundance of sulphur is a matter of debate. It is clear that an efficient solution to this problem could be reached making use of the measurements of the infrared lines of [SIV] - not yet available for a large sample of objects -.

The ionisation correction factor is a necessary step for the derivation of the empirical sulphur abundance calibration. It has been pointed out that a one-zone ionisation scheme may provide some insight into the situation for not ionisation bounded HII regions of moderate to low ionisation, where the various ions coexist throughout the nebula (e.g. Pagel 1978). For this reason we have presented above the general correlation between $\mathrm{S}^{+}+\mathrm{S}^{2+}$ and $\mathrm{S}_{23}$. A study of the ionisation correction factor scheme for sulphur is presented in the next section.

\section{The ICF scheme}

Perhaps one of the most difficult aspects in the derivation of total abundances is the question of the ionisation correction factor, ICF. The ICF for sulphur accounts for the contribution of the ionic species not detected in the optical. In high excitation 
Table 2. Sulphur abundances and values of $\log S_{23}$ for the sample of objects. Objects marked with ${ }^{a}\left({ }^{b}\right)$ have $S^{+}\left(S^{2+}\right)$ abundances derived using electron temperatures based on models from $t([\mathrm{OIII}])$. See text for details.

\begin{tabular}{|c|c|c|c|c|c|c|c|}
\hline Object & Ref. $^{a}$ & $12+\log \left(\frac{\mathrm{S}^{+}+\mathrm{S}^{2+}}{\mathrm{H}^{+}}\right)$ & $\log S_{23}$ & Object & Ref. $^{a}$ & $12+\log \left(\frac{\mathrm{S}^{+}+\mathrm{S}^{2+}}{\mathrm{H}^{+}}\right)$ & $\log S_{23}$ \\
\hline M 51 - CCM 10 & 1 & $6.95 \pm 0.10^{a}$ & $0.02 \pm 0.05$ & $\mathrm{~N} 4 \mathrm{~A}$ & 4 & $6.74^{a}$ & 0.02 \\
\hline M 51 - CCM53 & 1 & $6.95 \pm 0.14^{a}$ & $0.01 \pm 0.06$ & N79E & 4 & $6.59^{a, b}$ & 0.00 \\
\hline M 51 - CCM54 & 1 & $6.91 \pm 0.12^{a}$ & $0.08 \pm 0.05$ & N191A & 4 & $6.80^{a}$ & 0.14 \\
\hline M 51 - CCM55 & 1 & $7.04 \pm 0.11^{a}$ & $0.05 \pm 0.06$ & N80 & 4 & $6.02^{a, b}$ & -0.25 \\
\hline M 51 - CCM57 & 1 & $6.82 \pm 0.15^{a}$ & $-0.03 \pm 0.06$ & N83 & 4 & $6.24^{a, b}$ & -0.09 \\
\hline M 51 - CCM72 & 1 & $6.90 \pm 0.13^{a}$ & $-0.03 \pm 0.06$ & N13 & 4 & 6.20 & -0.08 \\
\hline NGC $1232-04$ & 2 & $6.43 \pm 0.21^{a}$ & $-0.05 \pm 0.08$ & N81 & 4 & $6.29^{b}$ & -0.07 \\
\hline NGC $1232-05$ & 2 & $7.01 \pm 0.16^{a}$ & $0.16 \pm 0.17$ & N66 & 4 & $6.01^{a, b}$ & -0.31 \\
\hline NGC $1232-07$ & 2 & $7.02 \pm 0.21^{a}$ & $0.08 \pm 0.06$ & NGC $604-\mathrm{A}$ & 5 & $6.82 \pm 0.03^{a, b}$ & $0.00 \pm 0.02$ \\
\hline NGC $1232-14$ & 2 & $6.84 \pm 0.16^{a}$ & $0.16 \pm 0.07$ & NGC $604-\mathrm{C}$ & 5 & $6.78 \pm 0.02^{a, b}$ & $0.09 \pm 0.03$ \\
\hline NGC $1365-05$ & 2 & $6.92 \pm 0.08^{a}$ & $0.08 \pm 0.01$ & NGC 604 - D & 5 & $7.03 \pm 0.13^{a}$ & $0.18 \pm 0.01$ \\
\hline NGC $1365-08$ & 2 & $6.81 \pm 0.08^{a}$ & $0.07 \pm 0.01$ & NGC $604-E$ & 5 & $7.00 \pm 0.14^{a}$ & $0.23 \pm 0.03$ \\
\hline NGC $1365-14$ & 2 & $6.96 \pm 0.11^{a}$ & $0.05 \pm 0.01$ & NGC 604 & 5 & $6.85 \pm 0.01^{a, b}$ & $0.06 \pm 0.01$ \\
\hline NGC $1365-15$ & 2 & $7.00 \pm 0.05^{a}$ & $0.23 \pm 0.01$ & NGC 604 & 8,5 & $6.79 \pm 0.09^{b}$ & $-0.01 \pm 0.07$ \\
\hline NGC $1365-16$ & 2 & $6.69 \pm 0.13^{a}$ & $0.03 \pm 0.01$ & NGC 604 & 8,22 & $6.66 \pm 0.16$ & $-0.01 \pm 0.07$ \\
\hline NGC $2997-04$ & 2 & $6.85 \pm 0.18^{a}$ & $0.18 \pm 0.08$ & NGC 604 & 8,33 & $6.68 \pm 0.09^{b}$ & $-0.01 \pm 0.07$ \\
\hline NGC $2997-05$ & 2 & $6.97 \pm 0.16^{a}$ & $0.18 \pm 0.08$ & NGC 595 & 8,33 & $6.78 \pm 0.15^{b}$ & $0.13 \pm 0.08$ \\
\hline NGC $2997-06$ & 2 & $6.92 \pm 0.16^{a}$ & $0.14 \pm 0.07$ & IC 131 & 8,22 & $6.50 \pm 0.18^{b}$ & $0.06 \pm 0.08$ \\
\hline NGC $2997-07$ & 2 & $6.92 \pm 0.17^{a}$ & $0.13 \pm 0.08$ & IC 131 & 8,33 & $6.65 \pm 0.15^{b}$ & $0.06 \pm 0.08$ \\
\hline NGC $2997-13$ & 2 & $7.10 \pm 0.14^{a}$ & $0.15 \pm 0.07$ & NGC 588 & 8,22 & $6.42 \pm 0.14^{b}$ & $0.08 \pm 0.07$ \\
\hline NGC $5236-03$ & 2 & $6.92 \pm 0.17^{a}$ & $0.12 \pm 0.07$ & NGC 588 & 8,33 & $6.63 \pm 0.09^{b}$ & $0.08 \pm 0.07$ \\
\hline NGC $5236-06$ & 2 & $7.00 \pm 0.20^{a}$ & $0.12 \pm 0.08$ & NGC 5471 & 8,24 & $6.18 \pm 0.13^{b}$ & $-0.17 \pm 0.07$ \\
\hline NGC $5236-11$ & 2 & $7.35 \pm 0.11^{a}$ & $0.30 \pm 0.07$ & IC $10-2$ & 8,23 & $6.61 \pm 0.06^{b}$ & $0.09 \pm 0.06$ \\
\hline NGC $5236-16$ & 2 & $7.25 \pm 0.13^{a}$ & $0.25 \pm 0.07$ & II Zw 40 & 8,7 & $6.13 \pm 0.05$ & $-0.22 \pm 0.06$ \\
\hline NGC $628-\mathrm{H} 13$ & 3 & $6.41 \pm 0.07^{a}$ & $-0.05 \pm 0.02$ & II Zw 40 & 8,14 & $6.06 \pm 0.12$ & $-0.22 \pm 0.06$ \\
\hline NGC 1232 - CDT1 & 3 & $7.13 \pm 0.16^{a}$ & $0.11 \pm 0.03$ & II Zw 40 & 8,21 & $6.11 \pm 0.06^{b}$ & $-0.22 \pm 0.06$ \\
\hline NGC 1232 - CDT2 & 3 & $6.52 \pm 0.16^{a}$ & $0.21 \pm 0.03$ & II Zw 40 & 8,23 & $6.14 \pm 0.06^{b}$ & $-0.22 \pm 0.06$ \\
\hline NGC 1232 - CDT3 & 3 & $6.92 \pm 0.09^{a}$ & $0.16 \pm 0.02$ & II Zw 40 & 8,24 & $6.12 \pm 0.06^{b}$ & $-0.22 \pm 0.06$ \\
\hline NGC 1232 - CDT4 & 3 & $6.93 \pm 0.07^{a}$ & $0.16 \pm 0.02$ & NGC 4861 & 8,6 & $6.37 \pm 0.12$ & $-0.02 \pm 0.12$ \\
\hline NGC 2467 & 4 & $6.39^{b}$ & -0.03 & NGC 2363 & 8,26 & $5.83 \pm 0.14$ & $-0.40 \pm 0.13$ \\
\hline$\eta \mathrm{Car}$ & 4 & 6.99 & 0.19 & I Zw 123 & 8,7 & $5.92 \pm 0.16^{b}$ & $-0.27 \pm 0.15$ \\
\hline M 17 & 4 & $6.92^{b}$ & 0.20 & Mrk 36 & 8,15 & $5.91 \pm 0.17^{b}$ & $-0.19 \pm 0.17$ \\
\hline M 20 & 4 & $6.99^{a}$ & 0.20 & Mrk 36 & 8,7 & $5.88 \pm 0.14^{b}$ & $-0.19 \pm 0.17$ \\
\hline NGC 3576 & 4 & $7.14^{b}$ & 0.46 & Mrk 600 & 8,24 & $6.00 \pm 0.14^{b}$ & $-0.34 \pm 0.13$ \\
\hline Orion 1 & 4 & 6.93 & 0.03 & I Zw 18 & 8,23 & $5.49 \pm 0.15^{a, b}$ & $-0.68 \pm 0.18$ \\
\hline Orion 2 & 4 & $6.97^{b}$ & 0.26 & I Zw 18 & 8,24 & $5.51 \pm 0.19^{a, b}$ & $-0.68 \pm 0.18$ \\
\hline N59A & 4 & $6.60^{a}$ & -0.01 & M 101 - H681 & 9 & $6.06 \pm 0.09^{a, b}$ & $-0.20 \pm 0.07$ \\
\hline N44B & 4 & $6.84^{a}$ & 0.09 & M 51 - CCM 10 & 10 & $7.16 \pm 0.20^{a}$ & $0.20 \pm 0.05$ \\
\hline N55A & 4 & $6.88^{a}$ & 0.04 & M 51 - CCM72 & 10 & $7.56 \pm 0.02^{a}$ & $0.41 \pm 0.00$ \\
\hline N113D & 4 & $6.90^{a}$ & 0.25 & NGC 2403 - VS35 & 11 & $6.77 \pm 0.17$ & $0.10 \pm 0.03$ \\
\hline N127A & 4 & 6.83 & 0.12 & NGC 2403 - VS24 & 11 & $7.04 \pm 0.11$ & $0.10 \pm 0.04$ \\
\hline N159A & 4 & $6.53^{a, b}$ & 0.02 & NGC 2403 - VS38 & 11 & $6.87 \pm 0.09$ & $0.06 \pm 0.02$ \\
\hline $\mathrm{N} 214 \mathrm{C}$ & 4 & $6.75^{a}$ & 0.12 & NGC 2403 - VS44 & 11 & $6.63 \pm 0.07$ & $0.04 \pm 0.02$ \\
\hline
\end{tabular}


Table 2. continued.

\begin{tabular}{|c|c|c|c|c|c|c|c|}
\hline Object & $\operatorname{Ref}^{a}$ & $12+\log \left(\frac{\mathrm{S}^{+}+\mathrm{S}^{2+}}{\mathrm{H}^{+}}\right)$ & $\log S_{23}$ & Object & Ref. $^{a}$ & $12+\log \left(\frac{\mathrm{S}^{+}+\mathrm{S}^{2+}}{\mathrm{H}^{+}}\right)$ & $\log S_{23}$ \\
\hline NGC 2403 - VS51 & 11 & $6.75 \pm 0.17$ & $0.06 \pm 0.06$ & NGC $3310-\mathrm{M}$ & 25 & $6.85 \pm 0.01^{b}$ & $0.06 \pm 0.01$ \\
\hline NGC 2403 - VS3 & 11 & $6.62 \pm 0.04$ & $0.06 \pm 0.03$ & II Zw 40 & 27,7 & $6.06 \pm 0.04$ & $-0.28 \pm 0.02$ \\
\hline NGC 2403 - VS49 & 11 & $6.59 \pm 0.17$ & $0.06 \pm 0.05$ & II Zw 40 & 27,14 & $6.04 \pm 0.05$ & $-0.28 \pm 0.02$ \\
\hline NGC 2403 - VS48 & 11 & $6.60 \pm 0.26$ & $0.08 \pm 0.09$ & II Zw 40 & 27,21 & $6.06 \pm 0.04$ & $-0.28 \pm 0.02$ \\
\hline NGC 7714 - A & 12 & $6.81 \pm 0.05^{a}$ & $0.19 \pm 0.01$ & II Zw 40 & 27,23 & $6.10 \pm 0.04$ & $-0.28 \pm 0.02$ \\
\hline NGC 7714 - N110 & 12 & $6.99 \pm 0.11^{a}$ & $0.18 \pm 0.05$ & II Zw 40 & 27,24 & $6.06 \pm 0.04$ & $-0.28 \pm 0.02$ \\
\hline NGC 7714 - B & 12 & $6.31 \pm 0.06^{a}$ & $0.01 \pm 0.02$ & Mrk 5 & 27,15 & $6.19 \pm 0.09$ & $0.07 \pm 0.02$ \\
\hline NGC 7714 - C & 12 & $6.41 \pm 0.18^{a}$ & $0.00 \pm 0.09$ & SBS0749+568 & 27,17 & $6.04 \pm 0.11$ & $-0.17 \pm 0.04$ \\
\hline NGC $7714-\mathrm{N}$ & 12 & $6.95 \pm 0.11^{a}$ & $0.22 \pm 0.05$ & SBS0926+606 & 27,17 & $6.04 \pm 0.11$ & $-0.17 \pm 0.04$ \\
\hline NGC 2363 - A2 & 13 & $5.92 \pm 0.08$ & $-0.41 \pm 0.02$ & Mrk 709 & 27,30 & $5.97 \pm 0.10$ & $-0.04 \pm 0.02$ \\
\hline M 101 - H1013 & 18 & $7.12 \pm 0.07$ & $0.21 \pm 0.02$ & Mrk 22 & 27,16 & $6.12 \pm 0.11$ & $-0.11 \pm 0.05$ \\
\hline M $101-$ H1105 & 18 & $6.95 \pm 0.06$ & $0.18 \pm 0.02$ & Mrk 1434 & 27,17 & $5.76 \pm 0.08$ & $-0.38 \pm 0.03$ \\
\hline M $101-$ H1159 & 18 & $6.68 \pm 0.09$ & $0.10 \pm 0.03$ & Mrk 36 & 27,7 & $5.96 \pm 0.12$ & $-0.21 \pm 0.04$ \\
\hline M $101-\mathrm{H} 1170$ & 18 & $7.08 \pm 0.08$ & $0.36 \pm 0.03$ & Mrk 36 & 27,15 & $5.98 \pm 0.13$ & $-0.21 \pm 0.04$ \\
\hline M $101-$ H1176 & 18 & $6.82 \pm 0.06$ & $0.14 \pm 0.02$ & VIIZw403 & 27,17 & $6.10 \pm 0.11$ & $-0.27 \pm 0.04$ \\
\hline M $101-$ H1216 & 18 & $6.55 \pm 0.07$ & $0.01 \pm 0.03$ & UM461 & 27,15 & $5.74 \pm 0.06$ & $-0.35 \pm 0.02$ \\
\hline M $101-\mathrm{H} 128$ & 18 & $6.81 \pm 0.07$ & $0.11 \pm 0.02$ & UM462 & 27,15 & $6.13 \pm 0.10$ & $-0.13 \pm 0.04$ \\
\hline M $101-$ H143 & 18 & $6.73 \pm 0.09$ & $0.13 \pm 0.03$ & Mrk 209 & 27,17 & $5.94 \pm 0.10$ & $-0.28 \pm 0.04$ \\
\hline M 101 - H149 & 18 & $6.72 \pm 0.07$ & $0.12 \pm 0.02$ & $\mathrm{IZw} 18-\mathrm{SE}$ & 28 & $5.31 \pm 0.12^{b}$ & $-0.78 \pm 0.07$ \\
\hline M 101 - H336 (S5) & 18 & $7.10 \pm 0.09$ & $0.21 \pm 0.03$ & IZw18-NW & 28 & $5.13 \pm 0.10^{b}$ & $-0.93 \pm 0.08$ \\
\hline M 101 - H409 & 18 & $6.63 \pm 0.07$ & $0.08 \pm 0.03$ & UGC4483 & 29 & $5.77 \pm 0.13$ & $-0.39 \pm 0.05$ \\
\hline M 101 - H67 & 18 & $6.60 \pm 0.14$ & $0.07 \pm 0.03$ & $\mathrm{~N} 160-\mathrm{A} 1$ & 31 & $6.91 \pm 0.06$ & $0.17 \pm 0.03$ \\
\hline M 101 - NGC 5471A & 18 & $6.29 \pm 0.07$ & $-0.11 \pm 0.03$ & $\mathrm{~N} 160-\mathrm{A} 2$ & 31 & $6.86 \pm 0.07$ & $0.14 \pm 0.03$ \\
\hline M 101 - NGC 5471B & 18 & $6.31 \pm 0.08$ & $0.02 \pm 0.03$ & $\mathrm{~N} 159-5$ & 31 & $7.01 \pm 0.10$ & $0.22 \pm 0.03$ \\
\hline M 101 - NGC 5471C & 18 & $6.42 \pm 0.08$ & $-0.05 \pm 0.03$ & N157 - B & 31 & $6.87 \pm 0.09$ & $0.24 \pm 0.04$ \\
\hline M 101 - NGC 5471D & 18 & $6.51 \pm 0.10$ & $-0.02 \pm 0.03$ & 30 Dor -1 & 31 & $6.72 \pm 0.08$ & $0.06 \pm 0.02$ \\
\hline M 101 - S5 & 19 & $7.31 \pm 0.04$ & $0.31 \pm 0.00$ & 30 Dor -2 & 31 & $6.80 \pm 0.09$ & $0.11 \pm 0.02$ \\
\hline SDSS J0133+1342 & 20,34 & $5.58 \pm 0.13^{a}$ & $-0.42 \pm 0.03$ & 30 Dor -3 & 31 & $6.87 \pm 0.06$ & $0.14 \pm 0.02$ \\
\hline KUG 0203-100 & 20,34 & $6.02 \pm 0.04^{a}$ & $0.04 \pm 0.05$ & 30 Dor -4 & 31 & $6.79 \pm 0.08$ & $0.12 \pm 0.02$ \\
\hline HS $0822+3542$ & 20,34 & $5.27 \pm 0.19^{a}$ & $-0.59 \pm 0.01$ & N11 - A & 31 & $6.78 \pm 0.09$ & $0.10 \pm 0.03$ \\
\hline I Zw 18 - NW & 20,34 & $5.08 \pm 0.18^{a}$ & $-0.78 \pm 0.04$ & N83 - B & 31 & $6.82 \pm 0.07$ & $0.13 \pm 0.03$ \\
\hline I Zw 18 - SE & 20,34 & $5.19 \pm 0.23^{a}$ & $-0.73 \pm 0.06$ & N79 - A & 31 & $6.96 \pm 0.07$ & $0.19 \pm 0.02$ \\
\hline SBS $1102+606$ & 20,34 & $5.67 \pm 0.19^{a}$ & $-0.29 \pm 0.02$ & $\mathrm{~N} 4-\mathrm{A}$ & 31 & $6.79 \pm 0.07$ & $0.10 \pm 0.02$ \\
\hline A1116+517 & 20,34 & $5.49 \pm 0.31^{a}$ & $-0.40 \pm 0.09$ & N88 - A & 31 & $5.92 \pm 0.10$ & $-0.32 \pm 0.03$ \\
\hline SDSS J1121+0324 & 20,34 & $5.49 \pm 0.31^{a}$ & $-0.44 \pm 0.06$ & N66 & 31 & $6.23 \pm 0.15$ & $-0.08 \pm 0.05$ \\
\hline SDSS J1201+0211 & 20,34 & $5.28 \pm 0.18^{a}$ & $-0.58 \pm 0.02$ & N81 & 31 & $6.22 \pm 0.10$ & $-0.17 \pm 0.03$ \\
\hline CGCG 269-049 & 20,34 & $5.39 \pm 0.21^{a}$ & $-0.50 \pm 0.05$ & S209 & 32 & $5.76 \pm 0.20^{b}$ & $-0.35 \pm 0.07$ \\
\hline M 101 - NGC 5455 & 22 & $6.75 \pm 0.10^{a, b}$ & $0.14 \pm 0.05$ & S127 & 32 & $6.42 \pm 0.29^{b}$ & $-0.12 \pm 0.07$ \\
\hline M 101 - NGC 5471 & 22 & $6.31 \pm 0.10^{a, b}$ & $-0.06 \pm 0.05$ & S128 & 32 & $6.40 \pm 0.29^{b}$ & $-0.09 \pm 0.06$ \\
\hline NGC 3310 - A & 25 & $6.82 \pm 0.03^{a}$ & $0.00 \pm 0.02$ & MA2 & 33 & $6.94 \pm 0.34^{b}$ & $0.08 \pm 0.02$ \\
\hline NGC 3310 - B & 25 & $6.78 \pm 0.02^{b}$ & $0.09 \pm 0.03$ & NGC 604 & 33 & $6.76 \pm 0.03^{b}$ & $0.06 \pm 0.01$ \\
\hline NGC $3310-C$ & 25 & $7.03 \pm 0.13$ & $0.18 \pm 0.01$ & NGC 595 & 33 & $7.02 \pm 0.09^{b}$ & $0.36 \pm 0.02$ \\
\hline NGC 3310 - E & 25 & $7.00 \pm 0.14$ & $0.23 \pm 0.03$ & & & & \\
\hline
\end{tabular}

${ }^{a}$ References are: 1. Bresolin et al. (2004); 2. Bresolin et al. (2005); 3. Castellanos et al. (2002); 4. Dennefeld \& Stasinska (1983); 5. Díaz et al. (1987); 6. Dinnerstein \& Shields (1986); 7. French (1980); 8. Garnett (1989); 9. Garnett \& Kennicutt (1994); 10. Garnett et al. (2004); 11. Garnett et al. (1997); 12. González-Delgado et al. (1995); 13. González-Delgado et al. (1994); 14. Guseva et al. (2000); 15. Izotov \& Thuan (1998); 16. Izotov et al. (1994); 17. Izotov et al. (1997); 18. Kennicutt et al. (2003); 19. Kinkel \& Rosa (1994); 20. Kniazev et al. (2003); 21. Kunth \& Sargent (1983); 22. Kwitter \& Aller (1981); 23. Lequeux et al. (1979); 24. Pagel et al. (1992); 25. Pastoriza et al. (2003); 26. Peimbert et al. (1986); 27. Pérez-Montero \& Díaz (2003); 28. Skillman \& Kennicutt (1993); 29. Skillman et al. (1994); 30. Terlevich et al. (1991); 31. Vermeij et al. (2002); 32. Vílchez \& Esteban (1996); 33. Vílchez et al. (1988); 34. this work. 


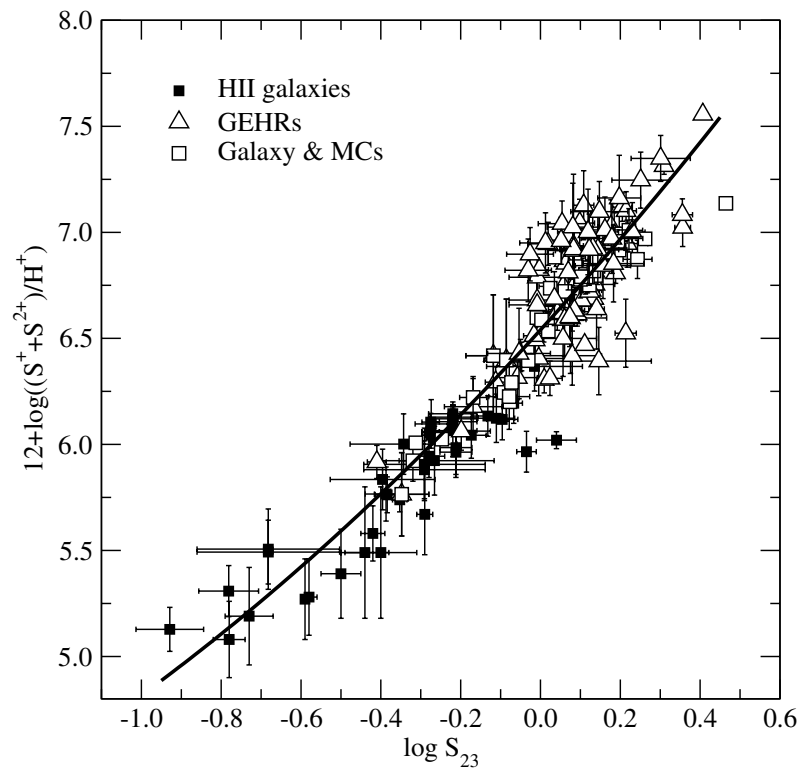

Fig. 1. The calculated sulphur abundance for the objects of the sample versus the $S_{23}$ parameter. The thick line represents the quadratic fit of the points.

nebulae a large fraction of the sulphur can be found in the $\mathrm{S}^{3+}$ stage, whose prominent emission lines of [SIV] are observed in the mid-IR at $10.52 \mu$. Therefore, in order to derive the total abundance of sulphur it is necessary to correct for the presence of $\mathrm{S}^{3+}$ in those objects for which there are no observations in the mid-IR, as follows:

$$
\begin{aligned}
\frac{N(\mathrm{~S})}{N(\mathrm{H})} & =\operatorname{ICF}\left(\mathrm{S}^{+}+\mathrm{S}^{2+}\right) \cdot \frac{N\left(\mathrm{~S}^{+}+\mathrm{S}^{2+}\right)}{N\left(\mathrm{H}^{+}\right)} \\
& =\frac{N\left(\mathrm{~S}^{+}+\mathrm{S}^{2+}+\mathrm{S}^{3+}\right)}{N\left(\mathrm{~S}^{+}+\mathrm{S}^{2+}\right)} \cdot \frac{N\left(\mathrm{~S}^{+}+\mathrm{S}^{2+}\right)}{N\left(\mathrm{H}^{+}\right)} .
\end{aligned}
$$

The first proposed ICF scheme for sulphur (Peimbert \& Costero 1969) was based on the similarity of the ionisation potentials of $\mathrm{O}^{+}(35.1 \mathrm{eV})$ and $\mathrm{S}^{2+}(34.8 \mathrm{eV})$

$\operatorname{ICF}\left(\mathrm{S}^{+}+\mathrm{S}^{2+}\right)=\frac{N(\mathrm{O})}{N\left(\mathrm{O}^{+}\right)}$

Nevertheless, some authors have pointed out that this relation has a strong correlation with the ionisation degree of the nebula (e.g. Barker 1978; Pagel 1978), thus implying an overestimation of the sulphur abundance in nebulae with low electron temperature. Barker (1980) proposed a new relation, based on the photoionisation models of Stasiǹska (1978):

$$
\operatorname{ICF}\left(\mathrm{S}^{+}+\mathrm{S}^{2+}\right)=\left[1-\left(1-\frac{N\left(\mathrm{O}^{+}\right)}{N(\mathrm{O})}\right)^{\alpha}\right]^{-1 / \alpha}
$$

for which he proposed a value of $\alpha=3$. The ICF from Peimbert $\&$ Costero corresponds to a value for $\alpha=1$ in this expression. Later, Izotov et al. (1994), based on photoionisation models from Stasiǹska (1990) gave a fit for this ICF that is quite similar to the Barker formula for $\alpha=2$.

In Fig. 2 we show our computation of the $\operatorname{ICF}\left(\mathrm{S}^{+}+\mathrm{S}^{2+}\right)$ as a function of $\log \left(\mathrm{O}^{+} / \mathrm{O}\right)$ using the data listed in Table 3 ,
Table 3. Abundances of $\mathrm{S}^{3+}$ for the objects for which there are ISO observations of the [SIV] $10.52 \mu$ emission line and the corresponding $\operatorname{ICF}\left(\mathrm{S}^{+}+\mathrm{S}^{2+}\right)$.

\begin{tabular}{cccc}
\hline \hline Object & Ref. $^{a}$ & $12+\log \left(\frac{\mathrm{S}^{3+}}{\mathrm{H}^{+}}\right)$ & $\mathrm{ICF}\left(\mathrm{S}^{+}+\mathrm{S}^{2+}\right)$ \\
\hline Mrk 209 & 1 & $5.93 \pm 0.20$ & $1.97 \pm 0.62$ \\
Mrk 209 & 1 & $5.68 \pm 0.23$ & $1.56 \pm 0.76$ \\
N160-A1 & 2 & $6.08 \pm 0.11$ & $1.15 \pm 0.05$ \\
N160-A2 & 2 & $5.90 \pm 0.10$ & $1.11 \pm 0.03$ \\
N159-5 & 2 & $6.03 \pm 0.10$ & $1.10 \pm 0.04$ \\
N157-B & 2 & $5.54 \pm 0.12$ & $1.05 \pm 0.02$ \\
30Dor-1 & 2 & $6.16 \pm 0.11$ & $1.27 \pm 0.10$ \\
30Dor-2 & 2 & $6.24 \pm 0.11$ & $1.27 \pm 0.10$ \\
30Dor-3 & 2 & $6.16 \pm 0.10$ & $1.20 \pm 0.06$ \\
30Dor-4 & 2 & $6.15 \pm 0.10$ & $1.23 \pm 0.07$ \\
N83-B & 2 & $5.45 \pm 0.12$ & $1.04 \pm 0.02$ \\
N4-A & 2 & $6.17 \pm 0.15$ & $1.24 \pm 0.11$ \\
N88-A & 2 & $5.80 \pm 0.10$ & $1.75 \pm 0.27$ \\
N66 & 2 & $5.73 \pm 0.15$ & $1.32 \pm 0.19$ \\
N81 & 2 & $5.45 \pm 0.11$ & $1.17 \pm 0.07$ \\
\hline
\end{tabular}

${ }^{a}$ The corresponding references for the emission line data of [SIV] at $10.5 \mu$ are: 1 . Nollenberg et al. (2002); 2. Vermeij et al. (2002).

${ }^{b}$ Assumming a constant $\mathrm{S}^{3+} / \mathrm{S}^{2+}$ ratio from the [SIII] lines in the nearIR from PMD03.

for three different values of $\alpha=1,2,3$. It is apparent in this plot that most points are better matched for $\alpha$ values between 2 and 3. Only one point, corresponding to a HII galaxy (Mrk 209, solid square), shows an ICF for a value of $\alpha$ even lower than 2. Since the $\mathrm{S}^{2+}$ abundances obtained from the [SIII] line at $18.71 \mu$ from the ISO observations from Nollenberg et al. (2002) are much higher than those obtained from the near-IR [SIII] (PMD03), we have recalculated $\mathrm{S}^{3+}$ abundances assuming a constant $\mathrm{S}^{3+} / \mathrm{S}^{2+}$ ratio. This value is showed in Table 3 and is represented as an open square in Figs. 2 and 3. Although showing a large error bar, the new value lies within the zone of $\alpha$ between 2 and 3, in better agreement with the values predicted by photo-ionisation models (PMD03).

Since the relevant lines for sulphur abundance determination are in the red to near infrared range, we try to relate the ICF scheme of sulphur to the ionisation structure as seen in this wavelength range. This aproach has the extra advantage of reducing the effect of reddening in the line ratios currently used. In order to derive a new ICF scheme for sulphur based only on the red-to-near infrared information, we need to combine available data and compute new models for reference objects for which we know all the relevant ionic abundances of sulphur. We have computed this relation as shown in Fig. 3. In this plot, we present the $\operatorname{ICF}\left(\mathrm{S}^{+}+\mathrm{S}^{++}\right)$vs. $\log ([\mathrm{SIII}] /[\mathrm{SII}])$ predicted by photo-ionisation models using CoStar model atmospheres of different effective temperatures $(35 \mathrm{kK}, 40 \mathrm{kK}$, 50 kK, Pérez-Montero \& Díaz 2005) and HII galaxy models (Pérez-Montero \& Díaz 2004), together with all the observed points available. The ICF predicted by a model with a single-star of $T_{\text {eff }}=35 \mathrm{kK}$ always gives $I C F=1$ no 


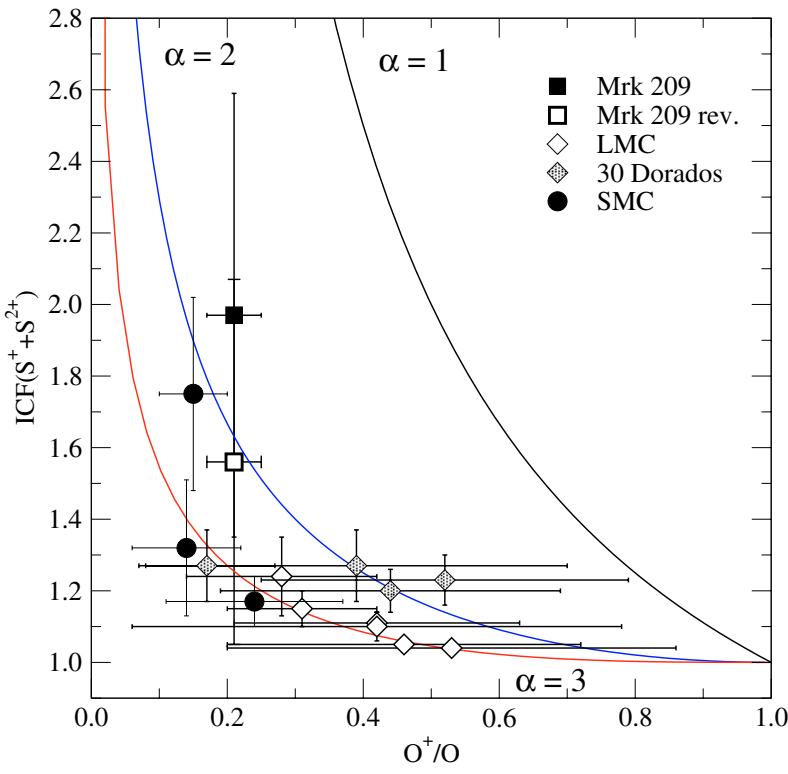

Fig. 2. Ionisation correction factor for the sum of $\mathrm{S}^{+}$and $\mathrm{S}^{2+}$ versus the $\mathrm{O}^{+} / \mathrm{O}$ ratio for the sample of objects listed in Table 3. The solid lines represent the Barker's formula for different values of the $\alpha$ parameter. Squares represent different values for Mrk 209 (see text for details), diamonds for HII regions in the Large Magellanic Cloud and circles in the Small Magellanic Cloud.

matter the excitation. The ICF predicted for objects presenting $\log ([\mathrm{SIII}] /[\mathrm{SII}]) \leq 0.2$ remains small, $1.0 \leq I C F \leq 1.05$. Above $\log ([\mathrm{SIII}] /[\mathrm{SII}])=0.4$, the ICF model predictions begin to diverge. This behaviour seems to be followed by the data. Giant HII region points cluster around the locus of $40 \mathrm{kK}$ models, except in the case of N88A, an SMC reddened, very young HII region breaking out of its natal cloud (Heydari-Malayeri et al. 1999). In this plot this giant HII region is located closed to Mrk 209, the only HII galaxy in Table 3. This indicates a hotter ionising source and a larger ICF. This behaviour could be consistent with the prediction from single burst evolutionary models (e.g. Stasińska et al. 2001) since HII galaxies typically host younger ionizing clusters than giant HII regions (Terlevich et al. 2004). Under this assumption, the equivalent width of $\mathrm{H} \alpha$, an age indicator, should provide a useful constraint.

\section{Discussion}

Earlier works by Christensen et al. (1997) and by Vermeij et al. (2002) have explored the abundance calibration of S/H versus $S_{23}$. Christensen et al. (1997) proposed a linear $S_{23}$ calibration as follows

$12+\log \left(\frac{\mathrm{S}}{\mathrm{H}}\right)=6.485+1.218 \log \mathrm{S}_{23}$

using data from giant HII regions and HII galaxies and complementary model predictions from Stasińska (1990). Although these points included some high metallicity HII regions of M 51 from Diaz et al. (1991), as well as lower metallicity objects from Garnett (1989), the whole abundance range was not

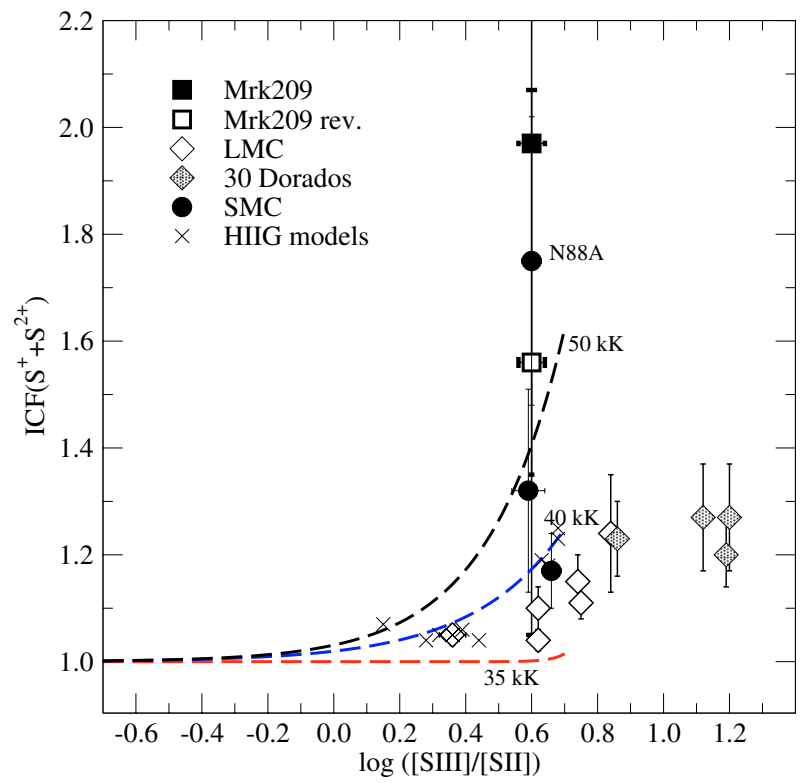

Fig. 3. Ionisation correction factor for the sum of $\mathrm{S}^{+}$and $\mathrm{S}^{2+}$ versus $\log ([\mathrm{SIII}] /[\mathrm{SII}])$ for the sample of objects listed in Table 3. The dashed lines represent the results from photo-ionisation models for CoStar single star atmospheres with the labelled effective temperature. Crosses represent results from photo-ionisation tailored models of HII galaxies. The rest of the symbols are the same as in Fig. 2.

sufficiently well sampled. They claimed that more data were needed before a definite conclusion could be drawn.

Vermeij et al. (2002) derived sulphur abundances for a new data set of optical and infrared spectra of HII regions in the Large and Small Magellanic Clouds. This information allowed these authors to derive all the ionic fractions of sulphur; including $\mathrm{S}^{+3}$ from the ISO [SIV] $10.5 \mu$ line for their sample objects. They present a comparison of their $\mathrm{S} / \mathrm{H}$ abundance points vs. $S_{23}$ and $S_{234}$ with model calculations for $\log \mathrm{U}=0$, $-1,-2$, and -3 . The following relation was found between $\log \mathrm{S}_{23}$ and $\log (\mathrm{S} / \mathrm{H})$

$\log \left(\frac{\mathrm{S}}{\mathrm{H}}\right)=-5.65+1.50 \log \mathrm{S}_{234}$

However, no relation was proposed for $\mathrm{S} / \mathrm{H}$ and $\mathrm{S}_{23}$.

In this work, we present an empirical abundance calibration, based on bright sulphur lines, which encompasses the whole range of abundance currently found in HII region studies. This calibration is firmly based on an extended homogeneous data base. In Fig. 4 we present the relation between the $\mathrm{S}_{23}$ parameter and the total abundance of sulphur for all the objects of our sample, taking into account the ionic abundances of $\mathrm{S}^{+}, \mathrm{S}^{2+}$ and the ICF corresponding to the formula of Barker for $\alpha=2.5$ which is the value that best fits the available points. The best quadratic fit to the data gives:

$12+\log \left(\frac{\mathrm{S}}{\mathrm{H}}\right)=6.622+1.860 \log \mathrm{S}_{23}+0.382\left(\log \mathrm{S}_{23}\right)^{2}$

with a dispersion of 0.185 dex in the range of $-1.0 \leq$ $\log \left(\mathrm{S}_{23}\right) \leq 0.5$. 


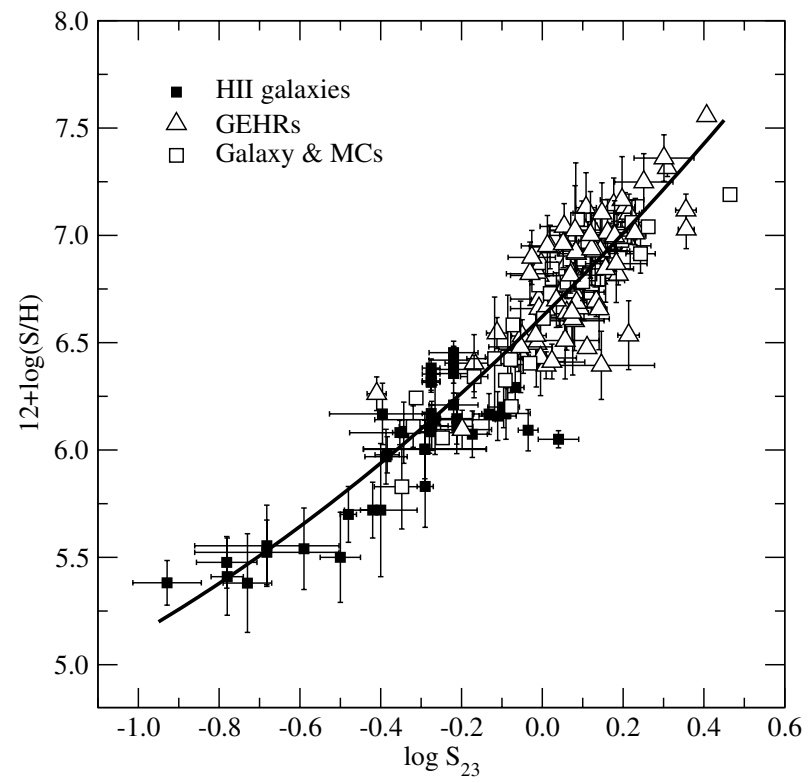

Fig. 4. Calculated total sulphur abundance for the objects of the sample versus the $S_{23}$ parameter. The thick line represents the empirical calibration proposed in this work.

This calibration is, to first order, independent of both $\mathrm{O} / \mathrm{H}$ and $\mathrm{O}_{23}$, and is especially suited to observations including only the red to near-infrared spectral ranges (from [SIII] $26312 \AA$, $\mathrm{H} \alpha$ up to the $1 \mu \mathrm{CCD}$ cut-off). Although the $\mathrm{S}_{23}$ parameter is possibly double valued, like $R_{23}$, the turnover region for $S_{23}$ is located above the range of sulphur abundances currently found in disk HII regions.

The calibration is, to some extent, affected by the ICF calculation scheme. However, only $34 \%$ of the objects in our sample show an ICF larger than 1.2 as derived from Barker's expression $(\alpha=2.5)$ and all of them show $\log ([\mathrm{SIII}] /[\mathrm{SII}])>0.4$, where model predictions are more uncertain. The calibration of $\left(\mathrm{S}^{+}+\mathrm{S}^{2+}\right) / \mathrm{H}^{+}$vs. $\mathrm{S}_{23}$ does not differ much from that of $\mathrm{S} / \mathrm{H}$ vs. $\mathrm{S}_{23}$ (see Figs. 1 and 4 ) and the values obtained from both for a given $S_{23}$ are within the quoted uncertainty in most cases.

This calibration makes possible the use of $\mathrm{S} / \mathrm{H}$ as a metallicity tracer in ionized nebulae. Its translation however to an $\mathrm{O} / \mathrm{H}$ abundance relies on the assumption that the $\mathrm{S} / \mathrm{O}$ ratio remains constant at all abundances. This point remains controversial (see e.g. Lodders 2003; Bresolin et al. 2004). Figure 5 shows the $\mathrm{S} / \mathrm{O}$ ratio vs. $12+\log (\mathrm{O} / \mathrm{H})$ for the objects in our sample. For all of them, the $\mathrm{O} / \mathrm{H}$ abundance has been calculated following the scheme presented in Pérez-Montero \& Díaz (2005). It can be seen from the figure that HII galaxy data are consistent with a constant $\mathrm{S} / \mathrm{O}$ ratio, but significantly lower than the solar ratio. Four galaxies deviate from this trend (UGC 4483, KUG 0203-100, Mrk 709 and SDSS J1121+0324). One of them, Mrk 709, also shows a large value of N/O (PMD03). Regarding disk HII regions the dispersion is much larger and the assumption of a constant $\mathrm{S} / \mathrm{O}$ is highly questionable.

Thus, following earlier work by Christensen et al. (1997) and Vermeij et al. (2002), we have derived an empirical calibration of the abundance of $\mathrm{S} / \mathrm{H}$ as a function of the $\mathrm{S}_{23}$

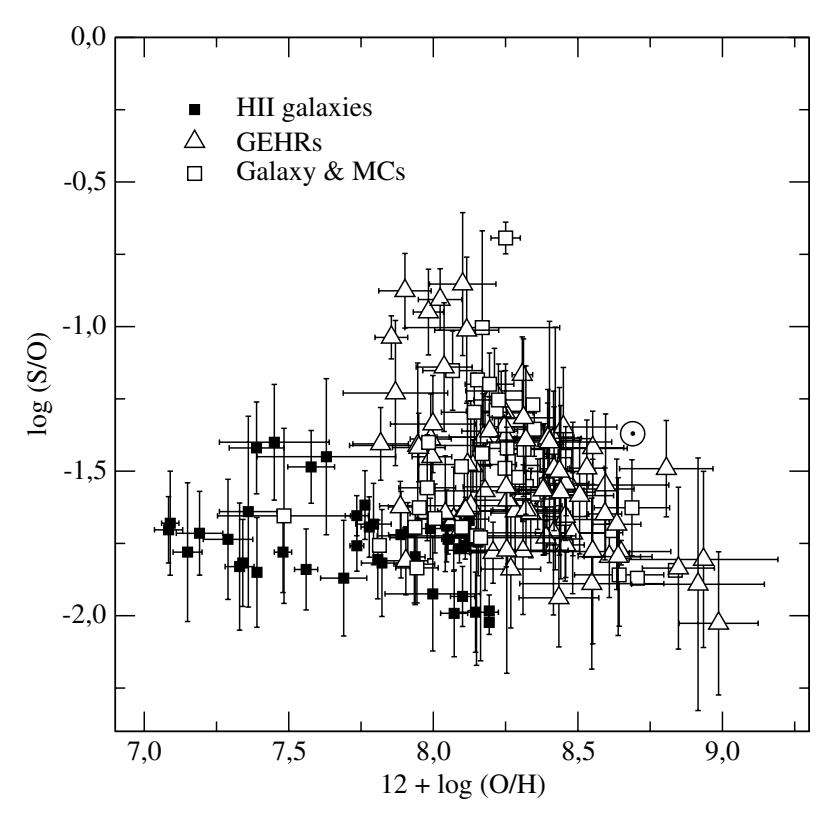

Fig. 5. Representation of the quotient of $\mathrm{S} / \mathrm{O}$ versus $\mathrm{O} / \mathrm{H}$ for the compiled sample and the solar value.

parameter, defined using bright sulphur lines, which we recommend as a useful tool to derive $\mathrm{S} / \mathrm{H}$ within a wide abundance range of 2 dex, keeping a statistical error of $0.18 \mathrm{dex}$ rms. This abundance range appears well suited to deal with objects from low metallicity HII galaxies to high metallicity HII regions located in the inner parts of the disks of spirals.

Acknowledgements. This work has been partially supported by projects AYA-2004-08260-C03-02 and AYA-2004-08260-C03-03 of the Spanish National Plan for Astronomy and Astrophysics.

\section{References}

Barker, T. 1978, ApJ, 219, 914

Barker, T. 1980, ApJ, 240, 99

Bresolin, F., Garnett, D. R., \& Kennicutt, R. C. 2004, ApJ, 615, 228

Bresolin, F., Schaerer, D., González-Delgado, R., \& Stasińska, G. 2005, A\&A, 441, 981

Castellanos, M., Díaz, A. I., \& Terlevich, E. 2002, MNRAS, 329, 315

Christensen, T., Petersen, L., \& Gammelgaard, P. 1997, A\&A, 322, 41

Dennefeld, M., \& Stasiǹska, G. 1983, A\&A, 118, 234

De Robertis, M. M., Dufour, R. J., \& Hunt, R. W. 1987, JRASC, 81, 195

Díaz, A. I., \& Pérez-Montero, E. 2000, MNRAS, 312, 130 (DPM00)

Díaz, A. I., Terlevich, E., Pagel, B. E. J., Vílchez, J. M., \& Edmunds, M. G. 1987, MNRAS, 226, 19

Díaz, A. I., Terlevich, E., Vílchez, J. M., Pagel, B. E. J., \& Edmunds, M. G. 1991, MNRAS, 253, 245

Dinerstein, H. L., \& Shields, G. A. 1986, ApJ, 311, 45

French, H. B. 1980, ApJ, 240, 41

Garnett, D. R. 1989, ApJ, 345, 282

Garnett, D. R. 1992, AJ, 103, 1330

Garnett, D. R., \& Kennicutt, R. C. 1994, ApJ, 426, 123

Garnett, D. R., Dufour, R. J., Peimbert, M., et al. 1995, ApJ, 449, 77

Garnett, D. R., Shields, G. A., Skillman, E. D., Sagan, S. P., \& Dufour, R. J. 1997, ApJ, 469, 93

Garnett, D. R., Kennicutt, R. C., \& Bresolin, F. 2004, ApJ, 607, L21 
González-Delgado, R. M., Pérez, E., Tenorio-Tagle, G., et al. 1994, ApJ, 437, 239

González-Delgado, R. M., Pérez, E., Díaz, A. I., et al. 1995, ApJ, 439, 604

Guseva, N. G., Izotov, Y. I., \& Thuan, T. X. 2000, ApJ, 531, 776

Heydari-Malayeri, M., Charmandaris, V., Deharveng, L., Rosa, M. R., \& Zinnecker, H. 1999, A\&A, 347, 841

Izotov, Y. L., \& Thuan, T. X. 1998, ApJ, 500, 188

Izotov, Y. L., Thuan, T. X., \& Lipovetsky, V. A. 1994, ApJ, 435, 647

Izotov, Y. L., Thuan, T. X., \& Lipovetsky, V. A. 1997, ApJS, 108, 11

Kennicutt, R. C., \& Garnett, D. R. 1996, ApJ, 456, 504

Kennicutt, R. C., Bresolin, F., French, H., \& Martin, P. 2000, ApJ, 537, 589

Kennicutt, R. C., Bresolin, F., \& Garnett, D. R. 2003, ApJ, 591, 801

Kinkel, U., \& Rosa, M. R. 1994, A\&A, 282, 37

Kniazev, A. Y., Grebel, E. K., Hao, L., et al. 2003, ApJ, 593, L73

Kunth, D., \& Sargent, W. L. W. 1983, ApJ, 273, 81

Kwitter, K. B., \& Aller, L. H. 1981, MNRAS, 195, 939

Lequeux, J., Rayo, J. F., Serrano, A., Peimbert, M., \& Torres-Peimbert, S. 1979, A\&A, 80, 155

Lodders, K. 2003, ApJ, 591, 1220

Nollenberg, J. G., Skillman, E. D., Garnett, D. R., \& Dinerstein, H. L. 2002, ApJ, 581, 1002

Oey, M. S., \& Shields, J. C. 2000, ApJ, 539, 687

Pagel, B. E. J., Edmunds, M. G., Blackwell, D. E., Chun, M. S., \& Smith, G. 1979, MNRAS, 189, 95

Pagel, B. E. J., Simonson, E. A., Terlevich, R. J., \& Edmunds, M. G. 1992, MNRAS, 255, 325
Pastoriza, M. G., Dottori, H. A., Terlevich, E., Terlevich, R., \& D'iaz, A. I. 1993, MNRAS, 260, 177

Peimbert, M., \& Costero, R. 1969, BAAS, 1, 256

Peimbert, A., Peimbert, M., \& Luridiana, V. 2002, ApJ, 565, 668

Peimbert, M., Peña, M., \& Torres-Peimbert, S. 1986, A\&A, 158, 266

Pérez-Montero, E., \& Díaz, A. I. 2003, MNRAS, 346, 105 (PMD03)

Pérez-Montero, E., \& Díaz, A. I. 2004, ASPC, 322, 213

Pérez-Montero, E., \& Díaz, A. I. 2005, MNRAS, 361, 1063

Saraph, H. E., \& Storey, P. J. 1999, A\&AS, 134, 369

Skillman, E. D., \& Kennicutt, R. C. 1993, ApJ, 411, 655

Skillman, E. D., Terlevich, R. J., Kennicutt, R. C. Jr., Garnett, D., \& Terlevich, E. 1994, ApJ, 431, 172

Skillman, E. D., Côté, S., \& Miller, B. W. 2003, AJ, 125, 593

Stasińska, G. 1978, A\&A, 66, 257

Stasińska, G. 1990, A\&AS, 83, 501

Stasińska, G., \& Leitherer, C. 1996, ApJS, 107, 661

Storey, P. J., \& Hummer, D. G. 1995, MNRAS, 272, 41

Tayal, S. S., \& Gupta, G. P. 1999, ApJ, 526, 541

Terlevich, R., Melnick, J., Masegosa, J., Moles, M., \& Copetti, M. V. F. 1991, A\&AS, 91, 285

Terlevich, R., Silich, S., Rosa-González, D., \& Terlevich, E. 2004, MNRAS, 348, 1191

Vermeij, R., Damour, J. M., van der Hulst, J. M., \& Baluteau, J.-P. 2002, A\&A, 390, 649

Vílchez, J. M., \& Esteban, C. 1996, MNRAS, 280, 720

Vílchez, J. M., Pagel, B. E. J., Díaz, A. I., Terlevich, E., \& Edmunds, M. G. 1988, MNRAS, 235, 633 